\title{
Power Considerations towards a Sustainable Pan-European Network
}

\author{
Anna Tzanakaki ${ }^{1}$, Kostas Katrinis ${ }^{*}{ }^{*}$, Tanya Politi ${ }^{2}$, Alex Stavdas ${ }^{2}$, Mario Pickavet ${ }^{3}$, Peter Van Daele ${ }^{3}$, \\ Dimitra Simeonidou ${ }^{4}$, Mike J. O’Mahony ${ }^{4}$, S. Aleksic ${ }^{5}$, Lena Wosinska ${ }^{6}$, Paolo Monti ${ }^{6}$ \\ Athens Information Technology (AIT), Peania, Greece, Email: atza@ait.edu.gr \\ University of Peloponnese.Email:\{tpoliti, astavdas\}@uop.gr \\ Ghent University - IBBT -IMEC,Email:\{ mario.pickavet, peter.vandaele\}@intec.ugent.be \\ University of Essex, Email:\{dsimeo,mikej\}@essex.ac.uk \\ Vienna University of Technology,Email: slavisa.aleksic@tuwien.ac.at \\ KTH - Royal Institute of Technology,Email:\{wosinska, pmonti\}@kth.se
}

\begin{abstract}
Energy savings are observed and quantified in the Pan-European network using transparent optical network technology. The network was dimensioned, using realistic traffic predictions of the optical networking roadmap of the European project BONE.

OCIS codes: (060.1155) All-optical networks, (060.4250) Networks
\end{abstract}

\begin{abstract}
1. Introduction
It has been shown that the ICT sector is responsible for about $4 \%$ of all primary energy today worldwide, and this percentage is expected to double by 2020 [1]. Network equipment plays a significant role in this consumption figures. Although several papers have been published to date addressing the issue of energy consumption in the ICT, and more specifically in optical transport networks, e.g. [2], [3], to the best of the authors' knowledge this paper is aiming for the first time at evaluating the energy savings that can be achieved through the use of wavelength routed optical transport networks in a realistic framework of the network of the future. The energy consumption results presented in this paper are obtained calculating the energy consumption of the optical network equipment required in a Pan-European telecommunications network as this is predicted by the European Optical Networking Roadmap. This roadmap is developed in the framework of the European Network of Excellence "Building the Future Optical Network in Europe” - BONE, funded by the European Union [4], [5].
\end{abstract}

\section{Traffic Projection Methodology and Power consumption estimation}

Trying to follow a realistic approach in predicting the capacity required by the European Network of the Future, the BONE optical networking roadmap takes as input the access bandwidth as well as the services and applications available to the end users currently and their expected evolution in the next five and ten years on a per European country basis. The methodology of the roadmap development is as follows [5]: information on existing and emerging applications and services available to end users, broadband penetration, existing and upcoming access technologies was collected for various European countries through a detailed survey performed among various BONE partners. This information was then processed taking into consideration the relevant country level demographics. The digital European diversity was considered in the future prediction of service and technology deployment as well as the relative population percentage.

Based on a per-country input data, and taking into consideration the parameters described above, an analysis, the details of which can be found in [5], was performed to produce the traffic generated/terminated and supported by the various European countries under consideration. A percentage of this traffic is effectively fed and has to be supported by the Pan-European network. We assumed that $20 \%$ of the traffic that is generated within each country is fed and will be serviced by the Pan-European network. Based on this assumption a matrix was produced indicating the volume of traffic that will enter the Pan-European network and will be associated with each European country considered in the analysis. Each European country considered is represented by the corresponding capital as a source/destination node thus forming the topology of the optical transport network under study. The source/destination information, in combination with the traffic data associated with it, is then converted into a conventional traffic matrix to be used for the Pan-European network dimensioning that will follow. This traffic matrix was obtained from the in-/out-bound traffic projections per source/destination site, assuming wavelength granularity of demands and uniform distribution of traffic to and from each node to all other nodes. Since the goal of the dimensioning study is to capture future trends rather than specify exact quantitative requirements, we consider

\footnotetext{
* The author participated in this work while at AIT. Currently, he is with the Exascale Stream Computing Collaboratory, IBM Research, Ireland.
} 
only a subset of all potential European source/destination nodes of the core traffic. Specifically, the Pan-European reference network topology proposed by the COST 239 action was considered [6].

A WDM optical network architecture based on Wavelength Selective Switch (WSS) nodes without wavelength conversion is assumed, as it offers a scalable approach that can support the very high capacity requirements predicted for the near and more so the longer term future of the Pan-European network [5]. The switching technology assumed was Micro-Electrical Mechanical Systems (MEMS), while the input/output fibers of the OXC's are supporting a maximum of 40wavelengths each. To provide comparative results and conclusions regarding the energy consumption of transparent vs. optoelectronic solutions, two alternative OXC technology options are evaluated: one supporting a transparent optical network, where data remains in the optical domain while traversing the optical path without optoelectronic conversions at any intermediate node and one that supports the opaque network architecture, whereby the optical signal is converted into the electronic domain at every intermediate node along each lightpath. The opaque OXC architecture is identical to the transparent one with the only difference being the presence of transponders surrounding the optical switch fabrics. Regarding the fiber links a model comprising a sequence of alternating Single Mode Fiber and Dispersion Compensation Fiber spans, is assumed. Optical amplifiers based on Erbium Doped Fiber Amplifier technology are allocated at the end of each transmission span to compensate for the associated insertion loss. The energy consumption figures used for all active devices are typical consumption figures originating from datasheet and surveys. Additionally, we incorporated power dissipation due to cooling in the power estimation mode used and a 100\% power overhead due to cooling is assumed [3].

\section{Network Dimensioning Framework}

The equipment energy consumption of the Pan-European network is calculated through a network dimensioning study that takes as input the predicted traffic demands produced by the BONE roadmap. The calculation of the dimensions of the European network is done for three different time periods and is performed by solving an instance of the network dimensioning problem referred to as "Brownfield Network Dimensioning". In this version of the problem, the geographical location of nodes is given together with the set of trenched physical links (ducts) connecting neighboring nodes. The output of the dimensioning process is the optimal number of fibers per link and wavelengths per fiber that need to be installed to serve the input traffic matrix at minimum cost, as well as the optimal dimensions of optical switches required to transparently route the input traffic. The adopted method employs a linear cost model incorporating fiber cost (fixed and length-dependent components), wavelength cost and sizedependent MEMS switch cost. In addition, all flow constraints are linear, thus admitting the formulation of the problem as a linear programming problem with integer solutions, similarly to the approach specified in [7]. Each source-destination demand (expressed at wavelength granularity) is served by $\mathrm{k}=3$ candidate lightpaths according to the standard k-shortest path routing, whereby physical distance is used for assigning link weights during the routing process. The results produced both from a network dimensioning and energy consumption perspective are of particular interest, as they are based on a realistic evaluation of the current and the future traffic demands that the Pan-European transport network has to support, generated through the BONE optical networking roadmap activity. For three input datasets of estimated aggregate traffic per site corresponding to three distinct time intervals, i.e., currently, in five years and in ten years from today, we generated traffic matrices assuming alternately 10Gb/s and $40 \mathrm{~Gb} / \mathrm{s}$ data rates per wavelength channel. Through this process, six input traffic matrices in total were obtained. Thus, forming 12 discrete network scenarios to be examined and compared with regards to the total power consumption. These scenarios are the following: current, near future (five years from today) and long term future (ten years from today) network supporting $10 \mathrm{~Gb} / \mathrm{s}$ per wavelength channel (switching granularity) for both cases the transparent and opaque OXC architectures and also current, near future (five years from today) and long term future (ten years from today) network supporting $40 \mathrm{~Gb} / \mathrm{s}$ per wavelength channel (switching granularity) for both cases the transparent and opaque OXC architectures.
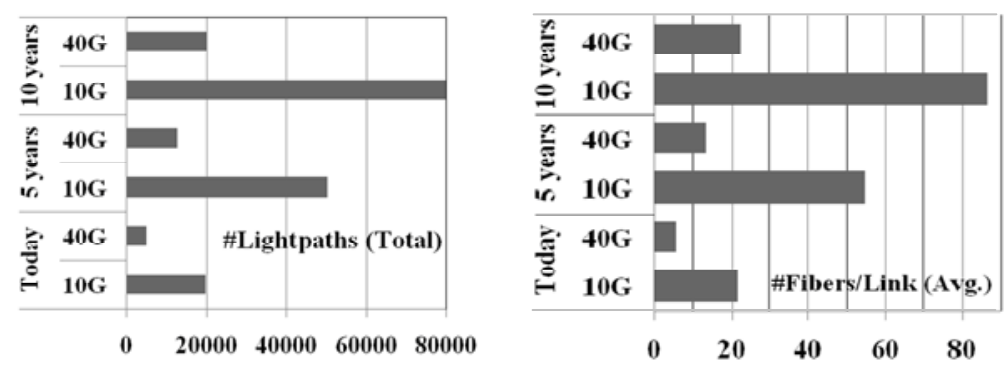

Figure 1. Metrics capturing the European optical backbone dimensions. 
Using the dimensioning method and the input parameter set outlined above, we created instances of Integer Linear Programs and solved each instance to optimality. This enabled us to specify the minimum-cost dimensions of the European optical core network corresponding to the present, as well as to its projected future evolution. Figure 1 depicts key metrics relevant to the evolution of the dimensions of the European backbone, including aggregate lightpaths number and average number of fibers installed per physical link. The migration to 40Gb/s per channel limits the dimensions of the optical network (in terms of node dimensions and number of fiber cables required) and reduces the incurred costs compared to the $10 \mathrm{~Gb} / \mathrm{s}$ case.

\section{Power Estimation Results and Discussion}

Based on the dimensions of the European optical network derived as described above the calculation of its present and future energy footprint is performed as a post-processing step. The results clearly indicate that the transparent approach offers significant energy savings compared to its optoelectronic counterpart. An evaluation of the benefits and limitations of different data rates supported on a wavelength basis with regards to energy efficiency was also carried out. More specifically Figure 2 (a), illustrates the total network power consumption for the two OXC architectures under study, supporting two possible per channel data rates i.e. $10 \mathrm{~Gb} / \mathrm{s}$ and $40 \mathrm{~Gb} / \mathrm{s}$. Power consumption results are produced for all three time periods under consideration. It is clear that the transparent approach is offering an energy saving that remains above 50\% compared to the opaque approach for all three periods and for the $10 \mathrm{~Gb} / \mathrm{s}$ per channel data rate case. The energy saving percentage obtained through the transparent approach is somewhat reduced for the $40 \mathrm{~Gb} / \mathrm{s}$ per channel data rate for which it remains above $33 \%$ for the current network case and increases to $40 \%$ for the near and longer term future network cases examined, that is a very significant saving. For both data rates, the energy savings observed are associated with the reduced number of transceiver requirements in the transparent case compared to the opaque case. To provide some more insight on the driving factors that determine the overall network power consumption, some additional results were produced (Figure 2 (b)). The results indicated that for both data rates the main power-dissipating elements are the transceivers followed by the EDFAs, while the least energy consuming elements are the optical switches. These conclusions can prove extremely useful in driving future efforts to reduce the energy footprint of optical technologies.

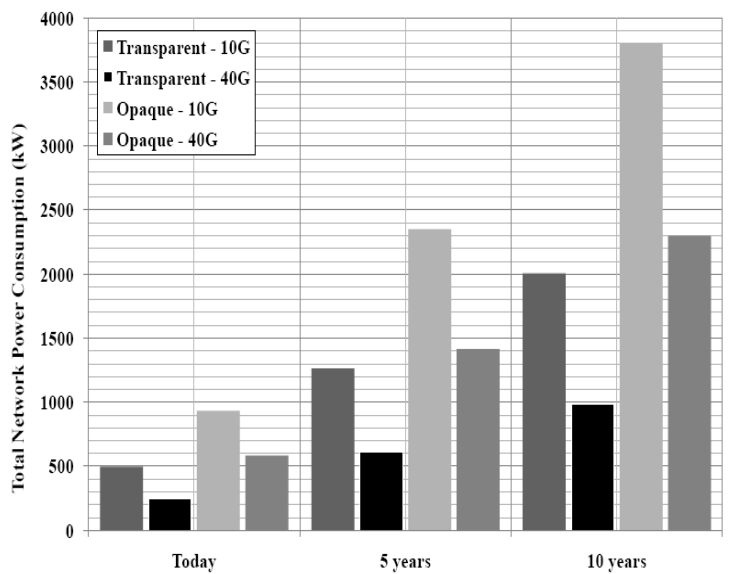

(a)

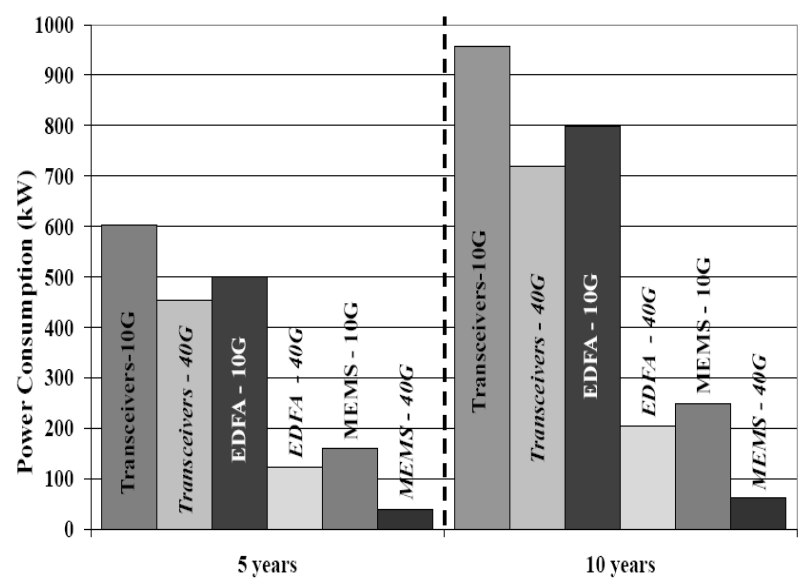

(b)

Figure 2 (a) Total network power consumption for $10 \mathrm{~Gb} / \mathrm{s}$ to $40 \mathrm{~Gb} / \mathrm{s}$ per wavelength, for the transparent and opaque architecture (b) Power consumption of the active network components for 10Gb/s and 40Gb/s per wavelength.

\section{Acknowledgement}

This work was performed under the support of the BONE-project ("Building the Future Optical Network in Europe"), a Network of Excellence funded by the European Commission in the 7th ICT- Framework Program.

\section{References}

[1] M. Pickavet et al., "Worldwide energy needs of ICT: the rise of power-aware networking”, IEEE ANTS, 2008.

[2] S. Aleksic, “Analysis of Power Consumption in Future High-Capacity Network Nodes”, IEEE/OSA JOCN, vol 1, n. 3, $245-258,2009$.

[3] J. Baliga et al., "Energy Consumption in Optical IP Networks", in IEEE/OSA JLT, vol. 27, no. 13, 2391-2403, 2009.

[4] http://www.ict-bone.eu/portal/landing_pages/index.html.

[5] C. Politi et al.,, "ICT BONE views on the Network of the Future: the role of optical networking”, ICTON $2010,2010$.

[6] P. Batchelor et al., "Study on the implementation of optical transparent transport networks in the European environment-Results of the research project COST 239”, Photonic Network Communications, vol. 2, no. 1, pp. 15-32, 2000.

[7] K. Katrinis et al., "Impairment Aware Network Design with Optimum Regenerator Placement”, NFOEC 2009, March 2009. 